\title{
INFLUÊNCIA DA CAMPANHA VACINAL CONTRA INFLUENZA SOBRE A MORBIMORTALIDADE DE IDOSOS POR DOENÇAS RESPIRATÓRIAS EM MINAS GERAIS, BRASIL
}

\author{
INFLUENCE OF VACCINATION CAMPAIGN AGAINST INFLUENZA ON THE MORBIMORTALITY OF \\ OLDER ADULTS BY RESPIRATORY DISEASES IN MINAS GERAIS, BRAZIL
}

\section{Carina Santos Maia ${ }^{a^{*}}$, Gisele Guedes Ruas Castanheira*, Lívia Cozer Montenegro*, Adriano Marçal Pimenta ${ }^{b^{*}}$}

amaiacarina.cm@gmail.com, badrianompimenta@yahoo.com.br

*Universidade Federal de Minas Gerais - Belo Horizonte (MG), Brasil

Data de recebimento do artigo: 10/12/2014 Data de aceite do artigo: 15/06/2015

\section{RESUMO}

Introduçáo: As doenças do aparelho respiratório e suas complicaçóes se destacaram nas últimas décadas, constituindo as principais causas de internação hospitalar na populaçáo maior de 60 anos. Objetivo: Analisar a influência da cobertura vacinal de idosos contra influenza nos indicadores de morbimortalidade por doenças respiratórias em Minas Gerais, Brasil, entre 1992 e 2010. Materiais e métodos: Estudo descritivo desenvolvido com dados de morbimortalidade e cobertura vacinal, consolidados pelo Departamento de Informática do Sistema Único de Saúde (DATASUS). Calculamos taxas de mortalidade, taxas de letalidade e frequência de internação. Resultados: Em alguns anos do período analisado, a cobertura vacinal estava abaixo do que se preconizava, porém próxima à meta estabelecida. A taxa de mortalidade por pneumonia para a faixa etária de 60 a 64 anos teve pouca alteraçáo durante o período estudado, entretanto, para os idosos de 65 anos ou mais, a taxa de mortalidade apresentou oscilaçáo. A taxa de letalidade foi crescente, ao longo do período, para as duas faixas de idade, sendo que para os idosos com 65 anos ou mais foi maior. Ao analisar a frequência de internaçáo por pneumonia, verificamos que em 1999 começou a diminuir. Contudo, para os idosos com 65 ou mais anos, a frequência de internaçáo voltou a subir em 2006, chegando ao maior patamar em 2010. Conclusáo: A vacinaçáo contra a influenza pode ter impacto na queda da morbimortalidade, mas ainda são necessárias estratégias de captação de idosos para que a adesão à vacina seja intensificada.

Palavras-chave: Doenças respiratórias; saúde do idoso; cobertura vacinal; imunização.

\section{ABSTRACT}

Introduction: Respiratory diseases and their complications have increased in recent decades and figured as the leading causes of hospitalization for the population aged over 60 years. Objective: To analyze the influence of influenza vaccination on the older adults' morbidity and mortality by respiratory diseases in Minas Gerais, Brazil, between 1992 and 2010. Materials and methods: Descriptive study conducted with morbimortality and vaccination coverage data, consolidated by the Departamento de Informática do Sistema Único de Saúde (DATASUS) [Data Processing Department of the Brazilian Unified Health System]. We calculated mortality, lethality, and hospitalization rates. Results: In some years of the analyzed period, the vaccination coverage was lower than the targeted, but it was close to it. The pneumonia mortality rate for the age group of 60-64 years had little change during the period. However, for the older adults with 65 years or more, the mortality rate shows considerable fluctuation. The lethality rate has increased over the period for both age groups, but was higher for the older adults with 65 years or more. By analyzing the frequency of pneumonia hospitalization during the period, it has been found that, in 1999, this frequency started decreasing, however, for the older adults with 65 years or more, in 2006, the frequency of hospitalization started to increase again, reaching its highest value in 2010. Conclusion: The 
vaccination against influenza might decrease the morbimortality, however there are still strategies necessary to attract the older adults with the focus of improving vaccination adherence.

Keywords: Respiratory tract diseases; health of the elderly; immunization coverage; immunization.

\section{Introdução}

Nas últimas décadas, o segmento composto por idosos vem crescendo significativamente. O envelhecimento da população mundial é um resultado do processo gradual de transição demográfica ${ }^{1}$. No Brasil, esse processo ocorre de forma brusca, veloz e em um contexto de grandes desigualdades sociais, constituindo-se em um desafio para o setor de saúde e outras esferas da sociedade $^{2}$. A Pesquisa Nacional por Amostra de Domicílio (PNAD) apontou que o percentual de pessoas com 60 anos ou mais era de 9,6\% em 2004, e, em 2012, subiu para $12,6 \%$. A projeção do Brasil, em 2025, é de que $15 \%$ a $20 \%$ da sua populaçáo total seja composta por indivíduos nessa faixa etária, ficando entre os 10 países do mundo com maior contingente de idosos ${ }^{3}$.

As transições demográficas e epidemiológicas da população trazem alteraçôes para a saúde, como as doenças crônicas que atingem a populaçáo idosa, as quais possuem peculiaridades ${ }^{4}$. As doenças crônicas mais comuns em idosos são as respiratórias, condiçóes coronárias avançadas, debilidade renal, doenças cardiovasculares, artrite, distúrbios emocionais ou psicológicos e doenças endócrinas, como a diabetes do tipo $\mathrm{II}^{5}$.

As doenças do aparelho respiratório (DAR) e suas complicaçôes se destacaram nas últimas décadas, sendo as principais causas de internação hospitalar na população maior de 60 anos, e mostrando-se mais graves nos portadores de doenças crônicas ${ }^{2,6}$

Portanto, a prevençáo da influenza tem impacto social e econômico importantes, pois leva à melhoria da qualidade de vida da populaçáo idosa e diminuição da internação hospitalar por doenças respiratórias. Nessa perspectiva, a principal intervenção preventiva para esse agravo é a vacinação ${ }^{2,3}$.

O Brasil iniciou as campanhas nacionais de vacinaçáo contra a gripe em 1999 e, desde entáo, a vacina é oferecida gratuitamente a pessoas com 65 anos ou mais de idade. A partir de 2000, a população de 60 a 64 anos também passa a ser beneficiada pela vacina ${ }^{4,7,8}$.

A maioria das pessoas infectadas pelo vírus da influenza se recupera no período de uma a duas semanas sem a necessidade de tratamento médico, e, por esse motivo, muitas vezes não é vista como uma ameaça ${ }^{4}$. No entanto, nas crianças muito pequenas, idosos e portadores de quadro clínicos especiais, a infecção pode levar a formas clinicamente graves, pneumonia e morte ${ }^{7}$.
Dessa forma, estudos referentes à influenza são importantes fontes de dados para auxiliar os gestores de serviços de saúde na criação de mecanismos que desenvolvam açóes de melhoria e cuidados para a população vulnerável às doenças respiratórias.

Este estudo teve como objetivo analisar a influência da cobertura vacinal de idosos contra influenza e indicadores de morbidade e mortalidade por doenças respiratórias em Minas Gerais nos anos de 1992 a 2010.

\section{Metodologia}

Trata-se de um estudo epidemiológico do tipo descritivo sobre a cobertura vacinal contra influenza e indicadores de morbimortalidade na população idosa do estado de Minas Gerais, durante o período de 1992 a 2010. Contemplou esse período para ser possível observar o comportamento das taxas utilizadas neste estudo antes e durante o início da campanha vacinal. Não foi possível estudar anos mais recentes, pois, até o momento da coleta de dados, não havia disponíveis informaçôes de cobertura vacinal e mortalidade, e, para os anos anteriores a 1992, não havia disponíveis dados referentes à morbidade.

Foram utilizados os dados de mortalidade e morbidade consolidados pelo Sistema de Informaçóes sobre Mortalidade (SIM) e Sistema de Informaçóes Hospitalares (SIH/SUS), gerenciados pelo Departamento de Informática do Sistema Único de Saúde (DATASUS) do Ministério da Saúde. As informações sobre cobertura vacinal em idosos e o quantitativo da população idosa também foram obtidos do DATASUS em sua seção sobre assistência à saúde e nos dados demográficos e socioeconômicos, respectivamente. Os dados foram transcritos em uma planilha Microsoft Excel, para viabilizar os cálculos necessários e pela melhor visualização das informaçôes obtidas.

Para o presente estudo, as faixas etárias utilizadas foram: 60 a 64 anos; 65 e mais anos, devido ao fato de no período entre 1999 e 2007 não haver um detalhamento de outras faixas etárias com dados referentes à vacinação. $\mathrm{O}$ estudo considerou essas duas faixas etárias pelo motivo de outras pesquisas mostrarem diferenças significativas entre elas ${ }^{8,9,10,11}$.

A doença do aparelho respiratório selecionada foi a pneumonia, sobre as quais as informaçóes de internação 
e óbito foram apresentadas por meio de frequências absolutas e relativas segundo as faixas etárias pré-estabelecidas e para cada ano de estudo (frequências de internação - número de internaçóes por uma determinada causa divido por todas as internaçóes, multiplicado por 100). Também foram calculadas as taxas de mortalidade (número de óbitos por determinada causa dividido pela população em risco, multiplicado por $10^{x}$ ) e de letalidade (número de óbitos por uma determinada causa dividida pelo total de internação por essa causa, multiplicada por 100).

O estudo avaliou a cobertura vacinal contra influenza para cada faixa etária, tendo em vista o cumprimento da meta estabelecida pelo Ministério da Saúde (até o ano de $2007 \geq 70 \%$ da populaçáo alvo; a partir de $2008 \geq 80 \%)^{7}$.

Este estudo foi realizado no contexto da Resolução $n^{\circ} 466 / 12$ do Conselho Nacional de Saúde que trata dos princípios éticos da pesquisa envolvendo seres humanos. Todavia, todos os dados utilizados foram obtidos em sistemas oficiais de informação de saúde, de domínio público, sem identificação individual e, portanto, sem possibilidade de haver infraçóes aos princípios éticos.

\section{Resultados}

No estudo realizado, levando-se em conta a cobertura para a população total, foi constatado que, até o ano de 2007 , apenas em 2000 a meta de vacinar $70 \%$ dos idosos não foi alcançada, cobrindo $64,5 \%$ da população-alvo. Nos anos seguintes, apenas em 2009 a meta de cobertura foi atingida, sendo superior a $80 \%$ da população-alvo, chegando a alcançar 84,7\% (Tabela 1).

A cobertura vacinal entre os idosos de 60 a 64 anos no primeiro ano da campanha de imunizaçáo contra a influenza (1999) foi baixa, apenas 4,7\%. Nos anos seguintes, a cobertura aumentou, porém, em 2000 $(57,2 \%)$ não alcançou a meta de vacinar $70 \%$ dos idosos. No período de 2001 a 2007, a meta foi alcançada, chegando a atingir 92,5\% dessa população em 2001. Quando a meta de vacinação passou para 80\% (2008), observou-se que apenas em 2009 conseguiu-se atingir tal objetivo (Tabela 1 ).

Para os idosos com idade igual ou maior a 65 anos, no primeiro ano da campanha, os resultados encontrados demonstram que $114,1 \%$ foram vacinados. Nessa faixa etária, nos anos de 2000 e 2001 (meta 70\%), 2008 e 2010 (meta 80\%), a meta não foi atingida (Tabela 1).

Tabela 1: Cobertura vacinal contra influenza (\%) em Minas Gerais, 1999 a 2010.

\begin{tabular}{ccccccc}
\hline Ano & \multicolumn{2}{c}{$\mathbf{6 0 - 6 4}$ anos } & \multicolumn{2}{c}{$\geq \mathbf{6 5}$ anos } & \multicolumn{2}{c}{ Total } \\
\hline & $\begin{array}{c}\text { Número de } \\
\text { vacinados }\end{array}$ & $\begin{array}{c}\text { Cobertura } \\
\text { vacinal }\end{array}$ & $\begin{array}{c}\text { Número de } \\
\text { vacinados }\end{array}$ & $\begin{array}{c}\text { Cobertura } \\
\text { vacinal }\end{array}$ & Número de vacinados & Cobertura vacinal \\
\hline 1999 & 22.058 & 4,7 & 1.105 .066 & 114,1 & 1.127 .124 & 78,4 \\
2000 & 294.425 & 57,2 & 753.984 & 67,9 & 1.048 .409 & 64,5 \\
2001 & 481.409 & 92,5 & 734.407 & 65,4 & 1.215 .816 & 74,01 \\
2002 & 443.617 & 84,3 & 845.557 & 74,6 & 1.289 .174 & 77,7 \\
2003 & 490.646 & 92,3 & 925.597 & 80,8 & 1.416 .243 & 84,5 \\
2004 & 511.636 & 95,3 & 1.003 .891 & 86,8 & 1.515 .527 & 89,5 \\
2005 & 485.629 & 88,5 & 1.020 .251 & 86,4 & 1.505 .880 & 87,1 \\
2006 & 481.952 & 86,8 & 1.049 .516 & 87,9 & 1.531 .468 & 87,6 \\
2007 & 484.415 & 78,3 & 1.077 .584 & 74,5 & 1.561 .999 & 75,6 \\
2008 & 478.418 & 74,1 & 1.161 .769 & 77,7 & 1.640 .187 & 76,6 \\
2009 & 560.058 & 83,2 & 1.317 .892 & 85,4 & 1.877 .950 & 84,7 \\
2010 & 568.833 & 79,5 & 1.260 .665 & 79,0 & 1.829 .498 & 79,2
\end{tabular}

Portanto, no período avaliado, a meta vacinal foi alcançada para a populaçáo como um todo em $75 \%$ (9/12), enquanto que para os idosos até 65 anos, esse desfecho foi verificado em $66,7 \%(8 / 12)$, e para os idosos com 65 e mais anos, 58,3\% (7/12).

Ademais, conforme verificado na Tabela 1 , existe uma variação na cobertura vacinal para a população como um todo e para ambas as faixas etárias.

Em relação à taxa de mortalidade por pneumonia, observa-se que, para o total da populaçáo, ela variou entre 14,0 a 21,3/10.000 (Tabela 2). Para a faixa etária de 60 a 64 anos, houve pouca alteração no período analisado, sendo a maior taxa igual a 5,3/10.000 em 1994 e, a menor, igual a 3,1/10.000 em 2002 (Tabela 2). Para os idosos de 65 anos ou mais, a taxa de mortalidade apresentou bastante oscilação tanto no período anterior à campanha vacinal contra a influenza quanto no período após o seu início, sendo a menor taxa no ano de $2000(18,8 / 10.000)$ e, a maior, no ano de 2010 $(29,2 / 10.000)$ (Tabela 2$)$. 
Tabela 2: Frequência absoluta de morte e taxa de mortalidade (por 10.000 pessoas na faixa etária) por pneumonia em Minas Gerais, 1992 a 2010.

\begin{tabular}{lcccccc}
\hline \multirow{2}{*}{ Ano } & \multicolumn{3}{c}{$\mathbf{6 0 - 6 4}$ anos } & \multicolumn{2}{c}{$\geq \mathbf{6 5}$ anos } & \multicolumn{2}{c}{ Total } \\
\cline { 2 - 7 } & $\mathbf{n}$ & Taxa & n & Taxa & n & Taxa \\
\hline $\mathbf{1 9 9 2}$ & 177 & 4,2 & 1.750 & 21,4 & 1.927 & 15,6 \\
$\mathbf{1 9 9 3}$ & 219 & 5,3 & 1.994 & 24,9 & 2.213 & 18,2 \\
$\mathbf{1 9 9 4}$ & 223 & 4,9 & 2.128 & 26,3 & 2.351 & 19,1 \\
$\mathbf{1 9 9 5}$ & 208 & 4,6 & 2.138 & 26,1 & 2.346 & 18,9 \\
$\mathbf{1 9 9 6}$ & 211 & 3,8 & 2.240 & 23,9 & 2.451 & 17,6 \\
$\mathbf{1 9 9 7}$ & 176 & 4,6 & 1.892 & 19,9 & 2.068 & 14,7 \\
$\mathbf{1 9 9 8}$ & 213 & 4,2 & 2.259 & 23,6 & 2.472 & 17,4 \\
$\mathbf{1 9 9 9}$ & 195 & 3,6 & 2.208 & 22,8 & 2.403 & 16,7 \\
$\mathbf{2 0 0 0}$ & 184 & 3,5 & 2.091 & 18,8 & 2.275 & 14,0 \\
$\mathbf{2 0 0 1}$ & 182 & 3,1 & 2.318 & 20,7 & 2.500 & 15,2 \\
$\mathbf{2 0 0 2}$ & 162 & 3,5 & 2.566 & 22,6 & 2.728 & 16,4 \\
$\mathbf{2 0 0 3}$ & 187 & 4,2 & 2.737 & 23,9 & 2.924 & 17,4 \\
$\mathbf{2 0 0 4}$ & 223 & 3,4 & 3.008 & 26,0 & 3.231 & 19,1 \\
$\mathbf{2 0 0 5}$ & 184 & 3,4 & 2.903 & 24,6 & 3.087 & 17,9 \\
$\mathbf{2 0 0 6}$ & 197 & 3,6 & 3.338 & 28,0 & 3.535 & 20,2 \\
$\mathbf{2 0 0 7}$ & 213 & 3,4 & 3.427 & 23,7 & 3.640 & 17,6 \\
$\mathbf{2 0 0 8}$ & 259 & 4,0 & 3.637 & 24,3 & 3.896 & 18,2 \\
$\mathbf{2 0 0 9}$ & 266 & 4,0 & 3.884 & 25,2 & 4.150 & 18,7 \\
$\mathbf{2 0 1 0}$ & 270 & 3,8 & 4.651 & 29,2 & 4.921 & 21,3 \\
\hline & & & & & &
\end{tabular}

A taxa média de mortalidade para os idosos de 60 a 64 anos, antes da implantação da vacina, foi igual a 4,7/10.000 e, para o período após o início da campanha vacinal, correspondeu a 3,7/10.000, mostrando, assim, uma queda de $21,3 \%$. Porém, para os idosos com idade igual ou maior que 65 anos, a taxa foi igual a 23,7/10.000 e $24,14 / 10.000$, no período antes e depois do início da campanha vacinal contra influenza, respectivamente, ou seja, praticamente permaneceu estável.

A Tabela 3 apresenta a taxa de letalidade por pneumonia na população no período estudado, a qual foi crescente ao longo do período para as duas faixas de idade, sendo mais elevada para os idosos com 65 ou mais anos.

Para a faixa etária de 60 a 64 anos de idade, a menor taxa de letalidade foi no ano de $1992(4,0 \%)$ e, a maior, no ano de 2008 (8,9\%). Para a faixa etária de 65 anos ou mais de idade, a menor e a maior taxa de letalidade corresponderam aos anos de 1993 (10,0\%) e 2010 $(17,5 \%)$, respectivamente.

Ao analisar a Tabela 4, constata-se que, no período de 1992 a 1998, a frequência de internação por pneumonia para idosos variou de 7,0\% a 8,3\%. A partir de 1999 , quando se iniciou a campanha vacinal contra a influenza, a

Tabela 3: Frequência absoluta de internação e taxa de letalidade (\%) por pneumonia em Minas Gerais, 1992 a 2010.

\begin{tabular}{|c|c|c|c|c|c|c|c|c|c|}
\hline \multirow{2}{*}{ Ano } & \multicolumn{3}{|c|}{ 60-64 anos } & \multicolumn{3}{|c|}{$\geq 65$ anos } & \multicolumn{3}{|c|}{ Total } \\
\hline & Internaçáo & Óbito & Taxa & Internaçáo & Óbito & Taxa & Internaçáo & Óbito & Taxa \\
\hline 1992 & 4.404 & 177 & 4,0 & 17.266 & 1.750 & 10,1 & 21.760 & 1.927 & 8,9 \\
\hline 1993 & 4.933 & 219 & 4,4 & 19.904 & 1.994 & 10,0 & 24.837 & 2.213 & 8,9 \\
\hline 1994 & 4.597 & 223 & 4,9 & 19.346 & 2.128 & 11,0 & 23.943 & 2.351 & 9,8 \\
\hline 1995 & 4.027 & 208 & 5,2 & 17.685 & 2.138 & 12,1 & 21.712 & 2.346 & 10,8 \\
\hline 1996 & 4.152 & 211 & 5,1 & 18.494 & 2.240 & 12,1 & 22.646 & 2.451 & 10,8 \\
\hline 1997 & 3.828 & 176 & 4,6 & 17.372 & 1.892 & 10,9 & 21.200 & 2.068 & 9,8 \\
\hline 1998 & 3.931 & 213 & 5,4 & 18.315 & 2.259 & 12,3 & 22.246 & 2.472 & 11,1 \\
\hline 1999 & 3.640 & 195 & 5,4 & 16.814 & 2.208 & 13,1 & 20.454 & 2.403 & 11,8 \\
\hline 2000 & 3.325 & 184 & 5,5 & 16.038 & 2.091 & 13,0 & 19.363 & 2.275 & 11,8 \\
\hline 2001 & 2.996 & 182 & 6,1 & 16.342 & 2.318 & 14,2 & 19.338 & 2.500 & 12,9 \\
\hline 2002 & 2.721 & 162 & 6,0 & 15.739 & 2.566 & 16,3 & 18.460 & 2.728 & 14,8 \\
\hline 2003 & 2.967 & 187 & 6,3 & 18.449 & 2.737 & 14,8 & 21.416 & 2.924 & 13,7 \\
\hline 2004 & 3.140 & 223 & 7,1 & 20.376 & 3.008 & 14,7 & 23.516 & 3.231 & 13,7 \\
\hline 2005 & 2.745 & 184 & 6,7 & 19.030 & 2.903 & 15,3 & 21.775 & 3.087 & 14,2 \\
\hline 2006 & 2.865 & 197 & 6,9 & 20.070 & 3.338 & 16,6 & 22.935 & 3.535 & 15,4 \\
\hline 2007 & 2.790 & 213 & 7,6 & 20.214 & 3.427 & 17,0 & 22.930 & 3.640 & 15,9 \\
\hline 2008 & 2.905 & 259 & 8,9 & 21.587 & 3.637 & 16,9 & 24.492 & 3.896 & 15,9 \\
\hline 2009 & 3.328 & 266 & 8,0 & 24.396 & 3.884 & 15,9 & 27.724 & 4.150 & 15,0 \\
\hline 2010 & 3.407 & 270 & 7,9 & 26.484 & 4.651 & 17,6 & 29.891 & 4.921 & 16,5 \\
\hline
\end{tabular}


frequência começou a cair em todas as faixas etárias, sendo 2002 o ano de menor número de internaçóes por essa causa.

Para a faixa etária de 60 a 64 anos, observou-se que, no ano de 2003, a frequência de internação começou a subir, tendo um pico em 2004 e outro em 2009. Porém, para os idosos com 65 ou mais anos, a frequência de internação teve um pico em 2004, diminuiu em 2005 e voltou a subir nos anos seguintes, observando-se, em 2010 a maior frequência de internaçóes no período analisado (Tabela 4).

\section{Discussão}

Os resultados deste estudo mostram que a meta vacinal contra influenza foi atingida para a população total na maior parte do período estudado. $\mathrm{Na}$ análise estratificada por faixas etárias o alcance da meta vacinal apresentou oscilaçóes, entretanto, também foi atingida na maioria dos anos (>65\%).

Com relação à mortalidade por pneumonia, apresentou diminuição apenas na faixa etária de 60 a 64 anos

Tabela 4: Frequência de internação por pneumonia (\%) em Minas Gerais, 1992 a 2010.

\begin{tabular}{|c|c|c|c|c|c|c|c|c|c|}
\hline \multirow[b]{2}{*}{ Ano } & \multicolumn{3}{|c|}{ 60-64 anos } & \multicolumn{3}{|c|}{$\geq 65$ anos } & \multicolumn{3}{|c|}{ Total } \\
\hline & $\begin{array}{c}\text { Internaçóes } \\
\text { por } \\
\text { pneumonia }\end{array}$ & $\begin{array}{c}\text { Total de } \\
\text { internaçóes } \\
\text { na faixa } \\
\text { etária }\end{array}$ & $\begin{array}{c}\text { Frequência } \\
\text { de } \\
\text { internaçáo }\end{array}$ & $\begin{array}{c}\text { Internaçóes } \\
\text { por } \\
\text { pneumonia }\end{array}$ & $\begin{array}{c}\text { Total de } \\
\text { internaçóes } \\
\text { na faixa } \\
\text { etária }\end{array}$ & $\begin{array}{c}\text { Frequência } \\
\text { de } \\
\text { internaçáo }\end{array}$ & $\begin{array}{c}\text { Internaçóes } \\
\text { por } \\
\text { pneumonia }\end{array}$ & $\begin{array}{c}\text { Total de } \\
\text { internaçóes } \\
\text { na faixa } \\
\text { etária }\end{array}$ & $\begin{array}{l}\text { Frequência } \\
\text { de } \\
\text { internaçáo }\end{array}$ \\
\hline 1992 & 4.404 & 74.286 & 5,9 & 17.266 & 236.238 & 7,3 & 21.760 & 310.524 & 7,0 \\
\hline 1993 & 4.933 & 76.882 & 6,4 & 19.904 & 248.889 & 8,0 & 24.837 & 325.771 & 7,6 \\
\hline 1994 & 4.597 & 73.535 & 6,3 & 19.346 & 243.374 & 8,0 & 23.943 & 316.909 & 7,6 \\
\hline 1995 & 4.027 & 63.215 & 6,4 & 17.685 & 220.793 & 8,0 & 21.712 & 284.008 & 7,6 \\
\hline 1996 & 4.152 & 59.937 & 6,9 & 18.494 & 214.430 & 8,6 & 22.646 & 274.367 & 8,3 \\
\hline 1997 & 3.828 & 59.250 & 6,5 & 17.372 & 214.859 & 8,1 & 21.200 & 274.109 & 7,7 \\
\hline 1998 & 3.931 & 56.215 & 7,0 & 18.315 & 209.387 & 8,8 & 22.246 & 265.602 & 8,4 \\
\hline 1999 & 3.640 & 55.923 & 6,5 & 16.814 & 202.748 & 8,3 & 20.454 & 258.671 & 7,9 \\
\hline 2000 & 3.325 & 55.439 & 6,0 & 16.038 & 202.003 & 7,9 & 19.363 & 257.442 & 7,5 \\
\hline 2001 & 2.996 & 54.464 & 5,5 & 16.342 & 208.595 & 7,8 & 19.338 & 263.059 & 7,4 \\
\hline 2002 & 2.721 & 55.259 & 4,9 & 15.739 & 218.383 & 7,2 & 18.460 & 273.642 & 6,8 \\
\hline 2003 & 2.967 & 56.105 & 5,3 & 18.449 & 224.901 & 8,2 & 21.416 & 281.006 & 7,6 \\
\hline 2004 & 3.140 & 54.476 & 5,8 & 20.376 & 219.579 & 9,3 & 23.516 & 274.055 & 8,6 \\
\hline 2005 & 2.745 & 52.972 & 5,2 & 19.030 & 219.811 & 8,7 & 21.775 & 272.783 & 8,0 \\
\hline 2006 & 2.865 & 53.013 & 5,4 & 20.070 & 222.689 & 9,0 & 22.935 & 275.702 & 8,3 \\
\hline 2007 & 2.790 & 53.120 & 5,3 & 20.214 & 220.977 & 9,2 & 22.930 & 274.097 & 8,4 \\
\hline 2008 & 2.905 & 54.313 & 5,4 & 21.587 & 216.989 & 10,0 & 24.492 & 271.302 & 9,0 \\
\hline 2009 & 3.328 & 54.745 & 6,1 & 24.396 & 223.862 & 10,9 & 27.724 & 278.607 & 10,0 \\
\hline 2010 & 3.407 & 58.419 & 5,8 & 26.484 & 234.640 & 11,3 & 29.891 & 293.059 & 10,2 \\
\hline
\end{tabular}

(4,7/10.000 em média para 3,1/10.000 em média). A letalidade apresentou perfil ascendente para ambas as faixas etárias. $\mathrm{O}$ mesmo padrão foi observado para a internação por pneumonia, apesar da tendência de queda para a faixa etária entre 60 e 64 anos.

Em relação à cobertura vacinal, foi observado para o ano de 1999 que os idosos de 60 a 64 anos tiveram uma baixa adesão à imunização. Tal fato pode ser explicado devido à campanha de imunização ter iniciado para essa faixa etária no ano de $2000^{7}$.

Constatou-se que, em alguns anos do período analisado, a cobertura vacinal estava abaixo, porém próxima da meta. Esse resultado também foi apresentado nos trabalhos de Lima-Costa ${ }^{8}$ e Francisco et al. ${ }^{12}$. Como observado por Francisco et al. ${ }^{13}$, uma das dificuldades em utilizar dados secundários é a flutuação na qualidade dos registros. Portanto, essa pode ser uma possível explicação para no ano de 1999 os idosos com 65 anos ou mais terem cobertura vacinal de $114,1 \%$. Outro motivo para o valor dessa cobertura poderia ser que idosos provenientes de outros estados vacinaram em Minas Gerais.

Outro aspecto interessante encontrado neste estudo foi a maior adesão à campanha vacinal por parte dos idosos entre 60 e 64 anos. Nos estudos de Lima-Costa ${ }^{8}$ e Francisco et al. ${ }^{9}$ foi observada maior adesão entre os idosos de 70 anos ou mais. Porém, uma limitação nesses estudos é que o status vacinal foi autorreferido, pois não foi possível confirmar a informação no cartão de vacina. Portanto, existe a possibilidade de ter ocorrido viés nas informações obtidas. 
Os resultados mostram que a taxa de mortalidade por pneumonia nos primeiros anos após o início da campanha vacinal foram as menores durante o período analisado. Para os anos seguintes, as taxas voltaram a ter valores similares aos anteriores à campanha. Tal resultado corrobora o estudo de Donalisio et al. ${ }^{6}$

A taxa de mortalidade apresentou oscilação para a faixa etária de 65 anos ou mais durante o período estudado e, para os idosos de 60 a 64 anos de idade, a taxa de mortalidade estabilizou em torno de 5 óbitos para cada 10.000 pessoas na faixa etária. Tendo em vista que as coberturas contra a influenza estão adequadas, uma hipótese plausível para essa oscilação seria o aumento da circulação de outros vírus de tropismo respiratório. Os mais frequentemente são: sindical respiratória, para influenza, adenovírus, rinovírus e metapneumovírus ${ }^{6,14}$.

$\mathrm{O}$ vírus da influenza apresenta variações antigênicas frequentes e aleatórias, do que decorre o aparecimento de novas variantes do vírus para os quais a população não apresenta imunidade, pois as cepas presentes na vacina podem não ser as mesmas que estáo circulando ${ }^{15}$.

No estudo de Campagna et al. ${ }^{10}$, que objetivou descrever a tendência das taxas de mortalidade por doenças respiratórias selecionas em idosos, observou-se aumento das taxas de mortalidade na populaçáo com 70 anos ou mais de idade e, para os idosos com 60 a 69 anos, a taxa de mortalidade se estabilizou. As autoras explicam que esse resultado pode ser devido às alteraçóes dos mecanismos de defesa, à redução da subnotificação de óbitos e proporção de óbitos notificados com causas mal definas de morte, mas que ainda se faz necessário investigar outras explicações para os resultados observados.

Obteve-se como resultado que a taxa de letalidade por pneumonia é crescente principalmente nos idosos mais velhos. Lundgren et al. ${ }^{11}$ constataram que a letalidade por pneumonia tem uma tendência ascendente, que pode ser justificada por internaçóes de casos mais graves de pneumonia e pelo envelhecimento da população. Verificaram, também, que a mortalidade por pneumonia varia de acordo com a idade, e que na última década aumentou nas faixas etárias superiores a 70 anos.

Malafaia ${ }^{16}$ e Scoralick et al. ${ }^{14}$ constataram que a efetividade da vacina contra influenza em indivíduos jovens é maior, conferindo de 65 a $90 \%$ de proteção contra o vírus causador da gripe. Em indivíduos idosos, devido ao progressivo declínio da função imune, o nível de proteção cai para uma porcentagem de 30\% a 50\%. A vacinação é benéfica na prevenção de morbidade e mortalidade por pneumonia, mas "há necessidade de estratégias de imunização que melhorem a eficiência das vacinas" 16 .

Ao analisar a frequência de internaçáo por pneumonia no período estudado (1992 a 2010), verificou-se que, no ano de 1999, ela começou a diminuir. Esse ano coincide com o início da campanha vacinal contra a influenza e, portanto, tal fato pode indicar que a vacinação produziu impacto positivo na prevenção de influenza ${ }^{2,13}$.

Outras hipóteses para explicar a diminuiçẫo da taxa de internaçáo hospitalar por doenças respiratórias em idosos nos anos de 1999 a 2002 são: a ampliação da rede ambulatorial, com maior acesso dos idosos aos serviços de saúde nos anos estudados e possível impacto na prevenção de internaçóes; a diminuição de leitos públicos e conveniados, dificultando o acesso às internaçóes, além da maior longevidade da população ${ }^{13}$.

Segundo Donalisio et al. ${ }^{6}$ e Daufenbach et al. ${ }^{17}, \mathrm{o}$ sistema de vigilância sentinela de quadros respiratórios registrou em 2003 e 2004 a circulação precoce do vírus da influenza antes da campanha vacinal. Tal circunstância pode ter contribuído para o aumento da frequência de internação por pneumonia nesses anos.

Em abril de 2009, a Organização Mundial de Saúde (OMS) declarou a Emergência em Saúde Pública de Importância Internacional devido a casos humanos de Influenza Suína (denominada Influenza A/H1N1) no México e nos Estados Unidos da América. Em julho do mesmo ano, foi constatado que o novo vírus estava circulando em território brasileiro ${ }^{18}$, o que seria uma provável explicação para que em 2009 tenha ocorrido outro pico de internaçóes por pneumonia em idosos de 60 a 64 anos em Minas Gerais.

Em relaçáo aos idosos com 65 anos ou mais de idade, verificou-se que a partir de 2003 a frequência de internação voltou a subir, tendo uma pequena queda em 2005 e voltando a subir nos anos seguintes. Ferrer et $\mathrm{al}^{2}{ }^{2}$ também chegaram a essa constatação e concluíram que o resultado pode ser devido ao número de leitos disponíveis e ao percentual de pessoas com 65 anos ou mais de idade na população.

Francisco et al. ${ }^{13}$ observaram que os idosos com idade mais avançadas apresentaram maiores indicadores proporcionais de hospitalizaçóes, e que os determinantes os quais podem estar associados a tal ocorrência são: a maior susceptibilidade imunológica, menores coberturas vacinais para esses idosos (foi detectado neste estudo que os idosos de 60 a 64 anos apresentaram maior adesão à campanha vacinal) e menor resposta imunológica à vacina contra a influenza.

\section{Conclusão}

No estudo realizado pode-se perceber que a adesão à campanha vacinal contra influenza atingiu patamares relevantes, principalmente para os idosos de 60 a 64 anos, grupo que apresentou queda na mortalidade por pneumonia após o início da campanha. Porém, a taxa 
de mortalidade de idosos com 65 anos ou mais praticamente náo se alterou, o que pode ser consequência da sua reduzida resposta imune à vacina e à sua menor adesão à imunização contra influenza em comparação aos que estão na faixa etária de 60 a 64 anos.

Foi observado que houve uma queda na frequência de internação logo após o início da campanha vacinal, sugerindo que a imunização pode ter produzido impacto positivo na prevençáo da influenza. Contudo, depois de 2003 a frequência na populaçáo com 65 anos ou mais volta a subir. Tal fato também pode ser explicado devido à menor adesão à campanha vacinal para essa faixa etária.

A vacinação contra a influenza pode ter impacto na queda da mortalidade e morbidade, mas ainda são necessárias estratégias de captação de idosos para que a adesão à vacina seja intensificada e sejam desenvolvidas vacinas com melhor eficiência na população em idade mais avançada.

A área da saúde é bastante minuciosa, e a busca pela excelência da qualidade dos serviços prestados é permanente. Sendo assim, os gestores de serviços de saúde enfrentam diversos desafios e têm que levar em conta todas as peculiaridades envolvidas. Para tanto, precisam estar informados e atualizados em relaçáo aos processos e resultados de programas para melhoria contínua dos serviços. É necessário que os gestores de serviços de saúde conheçam dados referentes à influenza para auxiliar na criação de ferramentas que desenvolvam açóes de melhoria e cuidados para a populaçáo vulnerável às doenças respiratórias.

\section{Referências}

1. Tannure MC, Alves M, Sena RR, Chianca TCM. Perfil epidemiológico da população idosa de Belo Horizonte, MG, Brasil. Rev bras Enferm. 2010;63(5):817-822. [acesso em: 20 out. 2013]. Disponível em: <http:// www.scielo.br/scielo.php?script=sci_arttext \&pi $\mathrm{d}=$ S0034-71672010000500020\&lng=en\&nrm=iso >

2. Ferrer ALM, Marcon SS, Santana RG. Morbidade hospitalar em idosos antes e após vacinação contra influenza no estado do Paraná. Rev Latino-Am. Enfermagem. 2008;16(5):1-6. [acesso em: 19 ago. 2013]. Disponível em: <http://www.scielo.br/scielo.php?script=sci_arttext\&pid=S0104-11692008000500006\&lng=en\&nrm=iso>

3. Geronutti DA, Molina AC, Lima SAM. Vacinaçáo de idosos contra a influenza em um centro de saúde escola do interior do estado de Sáo Paulo. Texto contexto - enferm. 2008;17(2):336-341. [acesso em: 19 ago. 2013]. Disponível em: <http://www.scielo.br/scielo.php?script=sci_arttext\&pid=S0104-07072008000200016\&lng=en\&nrm=iso >

4. Silva SPC, Menandro MCS. Representaçóes de idosos sobre a vacina da gripe. Ciênc saúde coletiva. 2013;18(8):21792188. [acesso em: 19 ago. 2013]. Disponível em: $<$ http://www.scielo.br/scielo.php?script=sci_arttext\&pi$\mathrm{d}=$ S1413-81232013000800002\&lng=en \&nrm =iso $>$. 5. Pinelli LAP, Montandon AAB, Boschi A, Fais LMG. Prevalência de doenças crônicas em pacientes geriátricos. Rev Odonto Ciênc.2005;20(47): 69-74. Disponível em: $<$ http://revistaseletronicas.pucrs.br/ojs/index.php/fo/article/viewFile/1153/912> [acesso em: 20 out. 2013].

5. Donalisio MR, Francisco PMSB, Latorre MRDO. Tendência da mortalidade por doenças respiratórias em idosos antes e depois das campanhas de vacinação contra influenza no Estado de São Paulo - 1980 a 2004. Rev bras epidemiol.2006;9(1):32-41. [acesso em: 19 ago. 2013]. Disponível em: <http://www.scielo.br/scielo.php?script=sci_arttext\&pid=S1415-790X2006000100006\&lng=en\&nrm=iso >

6. Campos EC, Sudan LCP, Mattos ED, Fidelis R. Fatores relacionados à vacinação contra a gripe em idosos: estudo transversal, Cambé, Paraná, Brasil. Cad Saúde Pública. 2012;28(5):878-888. [acesso em: 19 ago. 2013]. Disponível em: <http://www.scielosp.org/scielo.php?script=sci_arttext\&pid=S0102-311X2012000500007\&lng=en\&nrm=iso >

7. Lima-Costa MF. Fatores associados à vacinação contra gripe em idosos na região metropolitana de Belo Horizonte. Rev Saúde Pública. 2008;42(1):100-107. [acesso em: 19 ago. 2013].Disponível em: <http:// www.scielo.br/scielo.php?script $=s c i \_a r t t e x t \& p i-$ $\mathrm{d}=$ S0034-89102008000100013\&lng=en\&nrm=iso>

8. Francisco PMSB, Barros MBA, Cordeiro MRD. Vacinação contra influenza em idosos: prevalência, fatores associados e motivos da não-adesão em Campinas, São Paulo, Brasil. Cad. Saúde Pública. 2011;27(3):417426. [acesso em: 19 ago. 2013]. Disponível em: <http:// www.scielo.br/scielo.php?script $=$ sci_arttext\&pi$\mathrm{d}=$ S0102-311X2011000300003\&lng=en \&nrm=iso>

9. Campagna AS, Dourado I, Duarte EC, Daufenbach LZ. Mortalidade por causas relacionadas à influenza em idosos no Brasil, 1992 a 2005. Epidemiol Serv Saúde. 2009;18(3):209-218. [acesso em: 18 fev. 2014]. Disponível em: <http://scielo.iec.pa.gov.br/scielo.php?script=sci_arttext\&pid=S1679-49742009000300003\&lng=pt\&nrm=iso>

10. Lundgren F, Figueiredo MRF, Teixeira PJZ. Pneumonia adquirida na comunidade. In: Barreto SSM, Fiterman J, Lima MA. Prática Pneumológica.1a ed. Rio de Janeiro: Guanabara Koogan;2010. p. 212-225.

11. Francisco PMSB, Donalisio MR, Barros MBA, Cesar CLG, Garandina L, Goldbaum M. Vacinação contra influenza em idosos por área de residência: prevalência e fatores associados. Rev bras epidemiol. 2006;9(2):162171. [acesso em: 19 ago. 2013]. Disponível em: <http:// www.scielo.br/scielo.php?script $=$ sci_arttext \&pi$\mathrm{d}=$ S1415-790X2006000200003\&lng=en \& $\mathrm{nrm}=\mathrm{iso}>$

12. Francisco PMSB, Donalisio MR, Latorre MRDO. Internaçôes por doenças respiratórias em idosos e a 
intervenção vacinal contra influenza no Estado de São Paulo. Rev. bras. epidemiol. 2004;7(2):220227. [acesso em: 19 ago. 2013]. Disponível em:<http://www.scielo.br/scielo.php?script=sci_arttext\&pi$\mathrm{d}=$ S1415-790X2004000200011\&lng=pt\&nrm=iso >

13. Scoralick FM, Piazzolla LP, Pires LL, Neri C, Paula WK. Mortalidade por doenças respiratórias em idosos após campanhas vacinais contra influenza no Distrito Federal, Brasil, 1996-2009. J. bras pneumol. 2013:39(2):198204. [acesso em: 19 ago. 2013]. Disponível em: <http:// www.scielo.br/scielo.php?script $=$ sci_arttext $\&$ pi$\mathrm{d}=$ S1806-37132013000200011\&lng=en\&nrm=iso>

14. Forleo-Neto E, Halker E, Santos VJ, Paiva TM, Toniolo-Neto J.Influenza. Rev Soc Bras Med. Trop.2003;36(2): 267-274. [acesso em: 13 abr. 2014]. Disponível em:<http://www.scielo.br/scielo.php?script=sci_arttext\&pi$\mathrm{d}=$ S0037-86822003000200011\&lng=en \& $\mathrm{nrm}=$ iso>
15. Malafaia G. Implicaçóes da imunossenescência na vacinação de idosos. Rev Bras Geriatr Gerontol. 2008;11(3):433-441. [acesso em: 19 ago. 2013]. Disponível em: <http://www. crde-unati.uerj.br/img_tse/v11n3/capitulo10.pdf>

16. Daufenbach LZ, Carmo EH, Duarte EC, Campagna AS, Teles CAS. Morbidade hospitalar por causas relacionadas à influenza em idosos no Brasil, 1992 a 2006. Epidemiol Serv Saúde. 2009;18(1):29-44. [acesso em: 15 fev. 2014]. Disponível em: <http:// scielo.iec.pa.gov.br/scielo.php?script=sci_arttext\&pi$\mathrm{d}=$ S1679-49742009000100004\&lng=pt\&nrm=iso >

17. Milanest R, Caregnato RCA, Wachholz NIR. Pandemia de Influenza A (H1N1): mudança nos hábitos de saúde da populaçáo, Cachoeira do Sul, Rio Grande do Sul, Brasil, 2010. Cad Saúde Pública. 2011;27(4):723732. [acesso em: 18 fev. 2014]. Disponível em: <http:// www.scielo.br/scielo.php?script $=$ sci_arttext $\&$ pi$\mathrm{d}=$ S0102-311X2011000400011\&lng=en\&nrm=iso>

\section{Como citar este artigo:}

Maia CS, Castanheira GGR, Montenegro LC, Pimenta AM. Influência da campanha vacinal contra influenza sobre a morbimortalidade de idosos por doenças respiratórias em Minas Gerais, Brasil. Rev. Aten. Saúde. 2015;13(46):91-8 